\title{
Radiation Parameterization for Three-Dimensional Inhomogeneous Cirrus Clouds: Application to Climate Models
}

\author{
Y. Gu and K. N. Liou \\ Department of Atmospheric Sciences \\ University of California, Los Angeles \\ Los Angeles, California
}

\section{Introduction}

The effects of cirrus clouds on the radiation budget of the earth and the atmosphere, and hence their impact on weather and climate processes, have been articulated by Liou (1986, 1992). Cirrus clouds are frequently finite and highly inhomogeneous based on satellite and replicator sounding observations (Ou et al. 1995; Heymsfield and Miloshevich 1993). Potential effects of the cloud geometry and inhomogeneity on the transfer of radiation must be carefully studied to understand their impact on the radiative properties of the atmosphere as well as to perform proper interpretations of radiometric measurements from the ground, the air, and space. Most of the approaches to three-dimensional (3D) radiative transfer employ the Monte Carlo method. For application to cirrus clouds, Liou and Rao (1996) have used the successive orders of scattering (SOS) approach, which can be directly applied to specific geometry and inhomogeneous structure of a medium. Ou and Liou (1982) presented a spherical harmonic method in multiple dimensions, based on which the diffusion approximation for 3D radiative transfer can be developed (Liou 1992). However, the requirement of computer resources remains the primary obstacle in the modeling of 3D radiative transfer. In conjunction with our objective of understanding the effects of 3D inhomogeneous cirrus on radiative flux and heating rate profiles in the atmosphere and of providing a physical basis for parameterization in climate models, we have developed a 3D inhomogeneous radiative transfer model based on a modified diffusion approximation employing Cartesian coordinates.

\section{A 3D Inhomogeneous Radiative Transfer Model}

The general equation governing the transfer of diffuse intensity, I, can be expressed in the form

$$
-\frac{\mathrm{dI}(\mathrm{s}, \Omega)}{\beta_{\mathrm{e}}(\mathrm{s}) \mathrm{ds}}=\mathrm{I}(\mathrm{s}, \Omega)-\mathrm{J}(\mathrm{s}, \Omega)
$$

where $\mathrm{s}$ is the position vector; $\Omega$ is a unit vector representing the angular direction of scattering through the position vector; and $\beta_{\mathrm{e}}$ is the extinction coefficient for cloud particles. The source function, which is produced by the single scattering of the direct solar irradiance, multiple scattering of the diffuse intensity, and emission of the cloud, can be written as follows: 


$$
\mathrm{J}(\mathrm{s}, \Omega)=\frac{\varpi(\mathrm{s})}{4 \pi} \mathrm{P}\left(\mathrm{s} ; \Omega, \Omega_{0}\right) \mathrm{F}_{\bullet} \mathrm{e}^{-\tau_{\mathrm{s}}}+\frac{\varpi(\mathrm{s})}{4 \pi} \int_{4 \pi} \mathrm{I}\left(\mathrm{s}, \Omega^{\prime}\right) \mathrm{P}\left(\mathrm{s} ; \Omega, \Omega^{\prime}\right) \mathrm{d} \Omega^{\prime}+[1-\varpi(\mathrm{s})] \mathrm{B}(\mathrm{T}),
$$

where $\Phi$ is the single-scattering albedo; $\mathrm{P}$ is the phase function; $\mathrm{F}_{ }$is the incident solar irradiance; $\tau_{\mathrm{s}}$ is the optical depth in the direction of the incident solar beam; and $\mathrm{B}(\mathrm{T})$ is the Planck. Applicability of the source function to solar and thermal infrared regions is dependent on wavelength.

By expanding the phase function and the intensity in terms of spherical harmonic functions and by taking four terms in the expansion in a manner presented in Liou and Ou (1979) and Liou (1992), the following $3 \mathrm{D}$ inhomogeneous diffusion equation can be derived in the form

$$
\nabla \cdot\left(\nabla \mathrm{I}_{0}^{0} / \beta_{\mathrm{t}}\right)-3 \alpha_{\mathrm{t}} \mathrm{I}_{0}^{0}=\mathrm{F}_{\mathrm{t}}+\beta \Omega_{0} \cdot \nabla\left(\mathrm{F}_{\mathrm{t}} \mathrm{g} / \beta_{\mathrm{t}}\right)
$$

where $\beta_{\mathrm{t}}=\beta_{\mathrm{e}}(1-\bar{\varpi}), \alpha_{\mathrm{t}}=\beta_{\mathrm{e}}(1-\bar{\varpi})$, and

$$
F_{t}= \begin{cases}3 \beta_{\mathrm{e}} \mathrm{F}_{\mathrm{e}} \mathrm{e}^{-\tau_{\mathrm{s}}} / 4 \pi, & \text { Solar } \\ 3 \beta_{\mathrm{e}}(1-\bar{\omega}) \mathrm{B}(\mathrm{T}), & \mathrm{IR}\end{cases}
$$

In these equations, all the variables are functions of the coordinate $(\mathrm{x}, \mathrm{y}, \mathrm{z}) ; \mathrm{I}_{0}{ }^{0}$ is the first component of the intensity expansion; $\beta_{\mathrm{t}}$ and $\alpha_{\mathrm{t}}$ are terms associated with single-scattering properties; $\mathrm{g}$ is the asymmetry factor; $F_{t}$ is associated with the direct solar radiation and emission of cloud, respectively, depending on wavelength; and the last term in Eq. (3) vanishes for infrared (IR) bands.

With the diffusion approximation, the basic radiative transfer equation now transforms to a general second-order partial differential equation, in which all the coefficients are dependent on position vector. We have approached the solution of the system of equations by using a finite difference method. The central differential scheme with a second-order accuracy is employed for all partial differential terms in Eq. (3). The solution of the equation also requires the imposition of boundary conditions, which are set in such a manner that the incident diffuse flux at each surface is equal to zero or a constant. We use the successive overrelaxation (SOR) method to calculate $\mathrm{I}_{0}{ }^{0}$ at each grid point. Once $\mathrm{I}_{0}{ }^{0}$ is determined, the diffuse intensity and flux can then be obtained by

$$
\begin{gathered}
\mathrm{I}(\mathrm{x}, \mathrm{y}, \mathrm{z} ; \Omega)=\mathrm{I}_{0}^{0}(\mathrm{x}, \mathrm{y}, \mathrm{z})-\frac{3}{2 \mathrm{~h} \beta_{\mathrm{e}}} \sum_{\mathrm{i}=1}^{3} \frac{\partial \mathrm{I}_{0}^{0}(\mathrm{x}, \mathrm{y}, \mathrm{z})}{\partial \mathrm{x}_{\mathrm{i}}} \Omega_{\mathrm{x}_{\mathrm{i}}}+\frac{9 \mathrm{q}}{2 \mathrm{~h}}\left(\Omega \cdot \Omega_{0}\right) \mathrm{e}^{-\tau_{\mathrm{s}}}, \\
\mathrm{F}_{ \pm \mathrm{x}_{\mathrm{i}}}(\mathrm{x}, \mathrm{y}, \mathrm{z})=\int_{2 \pi} \mathrm{I}(\mathrm{x}, \mathrm{y}, \mathrm{z} ; \Omega) \Omega_{\mathrm{x}_{\mathrm{i}}} \mathrm{d} \Omega
\end{gathered}
$$

where $\mathrm{h}=3(1-\varpi \mathrm{g}) / 2 ; \mathrm{q}=\overline{\sigma g F}_{0} / 12 \pi, \Omega_{\mathrm{x}}=\left(1-\mu_{0}^{2}\right)^{1 / 2} \cos \phi, \Omega_{\mathrm{y}}=\left(1-\mu_{0}^{2}\right)^{1 / 2} \sin \phi$, and $\Omega_{\mathrm{z}}=\mu_{0}$. For thermal infrared radiation, the last term in Eq. (4) vanishes. The local rate of change of temperature is produced by the $3 \mathrm{D}$ radiative flux divergence in the form 


$$
\frac{\partial \mathrm{T}}{\partial \mathrm{t}}(\mathrm{x}, \mathrm{y}, \mathrm{z})=-\frac{1}{\mathrm{c}_{\mathrm{p}} \rho_{\mathrm{a}}} \nabla \cdot \mathrm{F},
$$

where $\mathrm{F}=\mathrm{iF}_{\mathrm{x}}+\mathrm{jF}_{\mathrm{y}}+\mathrm{kF}_{\mathrm{z}}$. To increase computational accuracy, we have applied the similarity principle

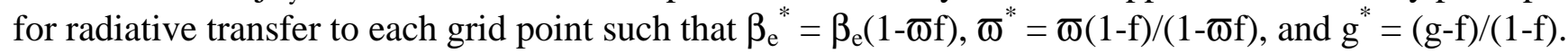
The fractional energy in the diffraction peak of the phase function $\mathrm{f}$ is taken to be $\bar{\varpi}_{4} / 9$, where $\varpi_{4}$ is the fourth moment in the phase function expansion. Calculations of the single-scattering properties are usually tedious and time consuming. For this reason, we follow the parameterization approach developed by $\mathrm{Fu}$ and Liou (1993) in determining the single-scattering properties.

To investigate the horizontal inhomogeneity, we use the optical depth and mean effective ice crystal size retrieved from the advanced very high-resolution radiometer (AVHRR) data. Moreover, the extinction coefficient varies in the vertical and can be estimated from the ice crystal data determined from the replicator sounding. By combining the satellite and replicator sounding data, a 3D ice water content (IWC) and mean effective ice crystal size field can be constructed (Liou and Rao 1996). For the homogeneous condition, mean single-scattering parameter values were used in the calculations. The solar zenith angle in this case is about $60^{\circ}$. Figure 1 displays the differences in the averaged heating rates in the $x-y$ and $y-z$ planes between inhomogeneous and homogeneous clouds. In the $x-y$ plane, the patterns correspond to the variabilities of the horizontal extinction coefficient (or IWC) field. More solar heating and IR warming are found in the area with larger extinction coefficients, while less solar heating and more IR cooling are shown in the area with smaller extinction coefficients. In the y-z plane, stronger IR cooling at the cloud top and a slightly more IR warming at the bottom are displayed in the inhomogeneous case. This is associated with smaller extinction coefficients in the upper part of the cloud and larger values in the lower part of the inhomogeneous cirrus cloud. For solar radiation, more heating is found in the inhomogeneous case in the whole $y-z$ plane.

\section{Application to Climate Models}

The formation of partial cloudiness and its consequence of vertical overlap appear to become a contemporary issue in the development of climate models. The present 3D radiative transfer model allows us to examine cloud overlapping effects on domain-averaged heating rates, an important parameter in climate models, defined by

$$
\overline{\left(\frac{\partial T}{\partial t}\right)}=\frac{1}{M N} \sum_{i=1}^{M} \sum_{j=1}^{N}\left(\frac{\partial T}{\partial t}\right)_{i, j},
$$

where $\mathrm{M}$ and $\mathrm{N}$ are the total grid points in the $\mathrm{x}$ and $\mathrm{y}$ directions, respectively. We may also investigate the effects of broken cloud fields on the radiative heating rate distribution. At the surface, we set a twodimensional surface albedo so that an upward solar flux reflected from the surface at the lower boundary can be taken into account. For the upper boundary, the direct solar flux at the top of atmosphere is attenuated to the position of the top model domain. A proper adjustment in clear sky is also performed by adding a background aerosol distribution, which represents a more realistic clear condition and, at the same time, the Lorenz-Mie type scattering will allow an adjustment of the photon free path length. 


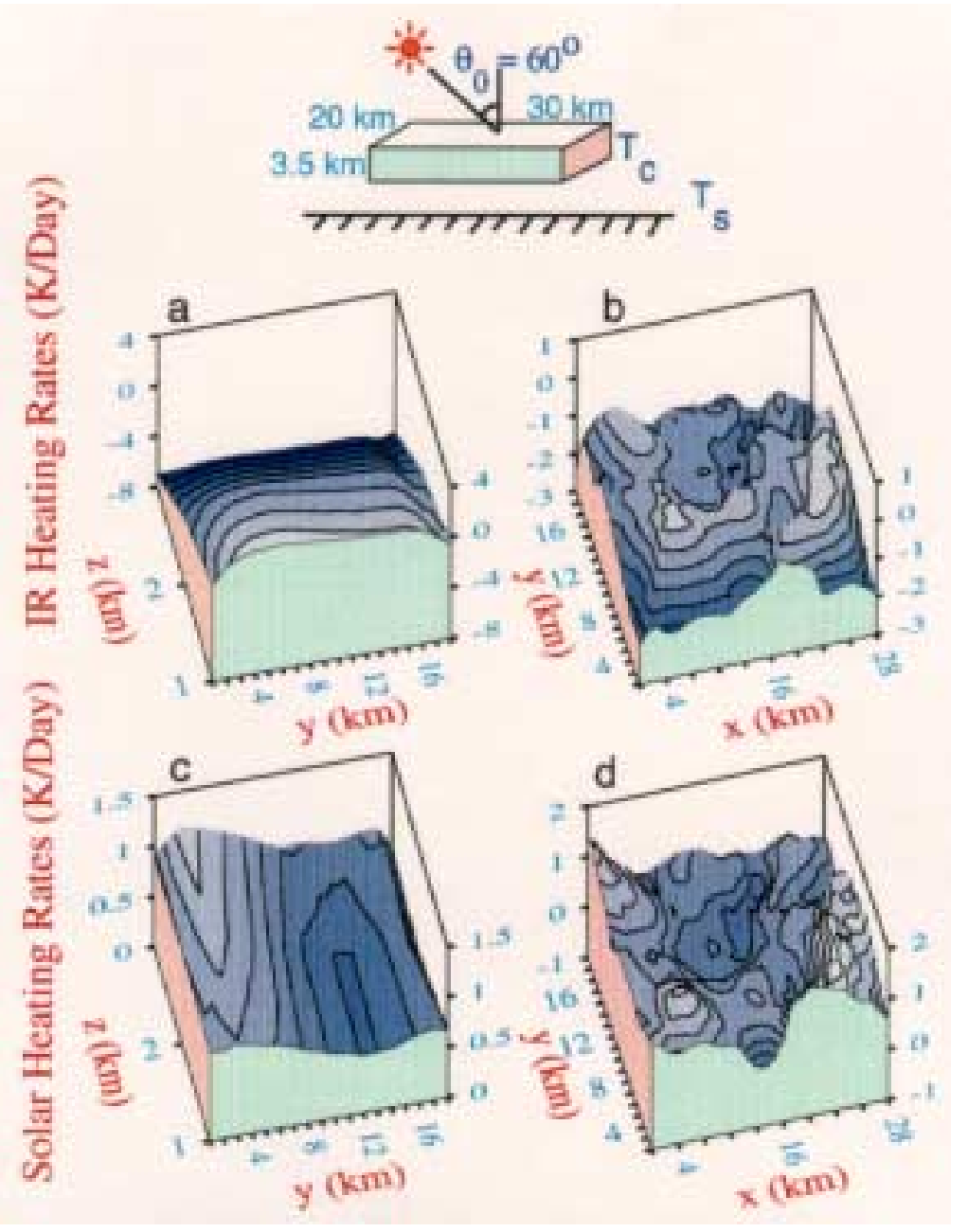

Figure 1. 3D images of the differences in IR ( $a$ and $b)$ and solar ( $c$ and $d)$ heating rates ( $\mathrm{k} /$ day) between inhomogeneous and homogeneous cirrus cloud layers.

Three experiments are designed for cloud overlap study, as shown in Figures 2a-c, which display a number of possible distributions for low, middle, and high clouds, leading to different numbers of columns. Two experiments designed to investigate broken cloud effects are shown in Figures $2 \mathrm{~d}$ and $2 \mathrm{e}$. Each cloud is considered to be homogeneous with a thickness of $1 \mathrm{~km}$ and a cloud fractional cover of $60 \%$. The middle- and low-level clouds are composed of liquid water, while the high-level cloud consists of ice particles. Figures $2 \mathrm{a}$ and $2 \mathrm{c}$ correspond to maximum (with a total cloud cover $\eta=60 \%$ ) and random $(\eta=97.6 \%)$ overlap, respectively, while Figure $2 \mathrm{~b}$ represents the cloud structure with maximum overlap for middle- and low-level clouds and random overlap between high cloud and the two combined cloud field $(\eta=88 \%)$. The solar zenith angle is taken to be $60^{\circ}$ in these calculations to highlight the 3D effect. 


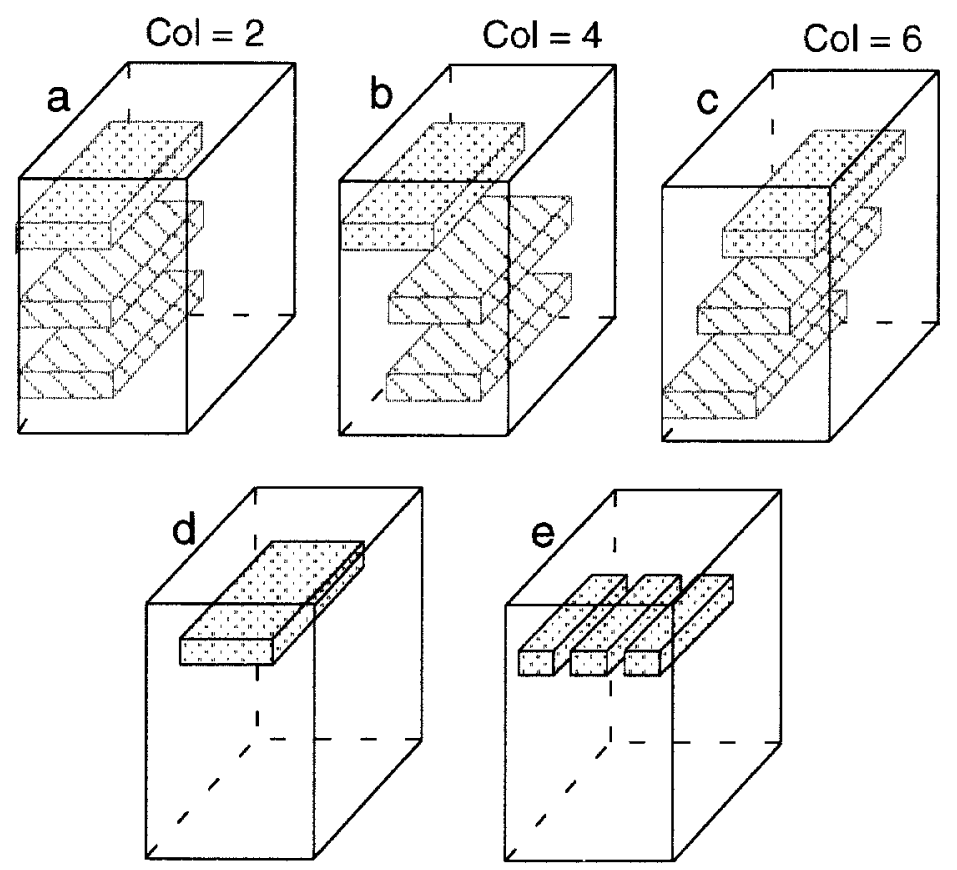

Figure 2. Potential cloud configurations generated from GCMs. Distributions of the distinct possible positions for high, middle, and low clouds are displayed in a-e: (a) two columns, (b) four columns, (c) six columns, (d) continuous cloud field, and (e) broken clouds.

Domain-averaged solar heating rates for each cloud overlap calculated from the PP method are shown in Figure 3a. The heating rate patterns for the three cloud configurations are in general agreement, except at the top of middle- and low-level clouds where maximum overlap produces less heating due to a smaller cloud cover. The heating profiles computed from the 3D model, however, show substantial differences (Figure 3b). Clouds with random overlap produce larger total cloud cover and consequently generate more heating than those with maximum overlap, especially for large solar zenith angle. Comparisons of the heating rate results between 3D and PP models are displayed in Figure 3c-e. The differences are more pronounced for larger solar zenith angles because photons can enter through cloud sides and be trapped within the cloud. It is interesting to note that while heating within the middle cloud is significantly greater for 4- and 6-column cloud configurations, heating results for 2-column, i.e., maximum overlap, are smaller than those from the PP case. Thus, how the clouds are vertically positioned is critically important in the determination of heating/cooling rates. This issue should receive more attention in future studies regarding the treatment of cloud vertical structure in climate models.

Compared to solar heating rates, domain-averaged IR heating/cooling rates show relatively smaller differences among different cloud configurations in both 3D and PP models. However, larger IR cooling is produced in the low cloud for random overlap configuration, while slightly smaller IR cooling is generated in the middle cloud for maximum overlap configuration in the PP case, primarily associated with cloud covers. Results from 3D and PP models show significant differences. 3D clouds produce 

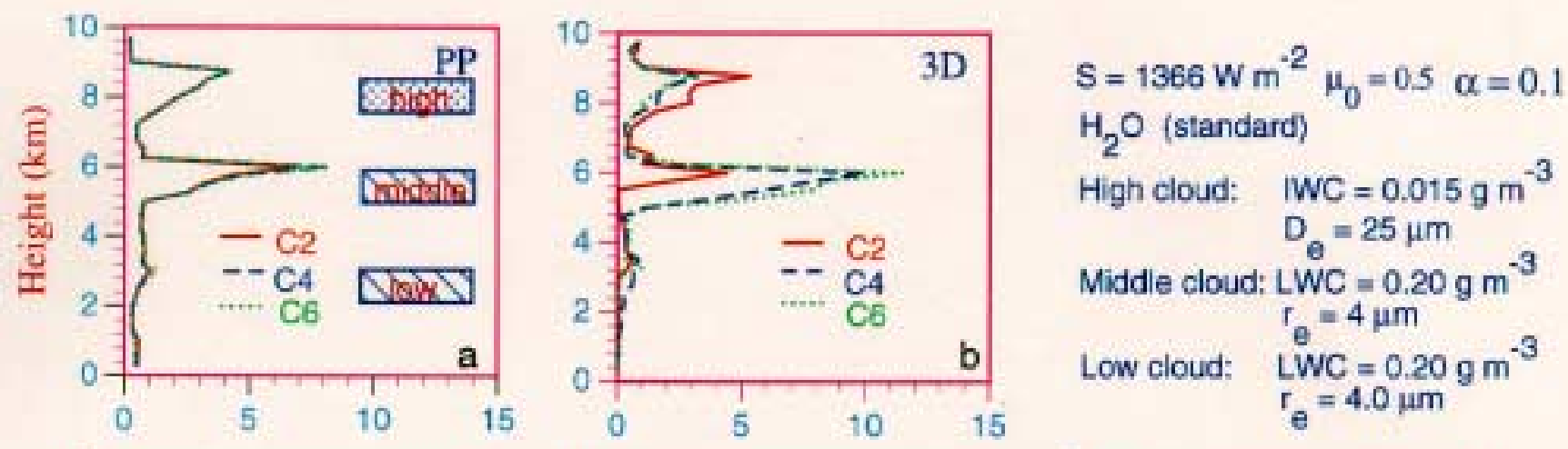

Middle cloud: $\mathrm{LWC}=0.20 \mathrm{~g} \mathrm{~m}^{-3}$
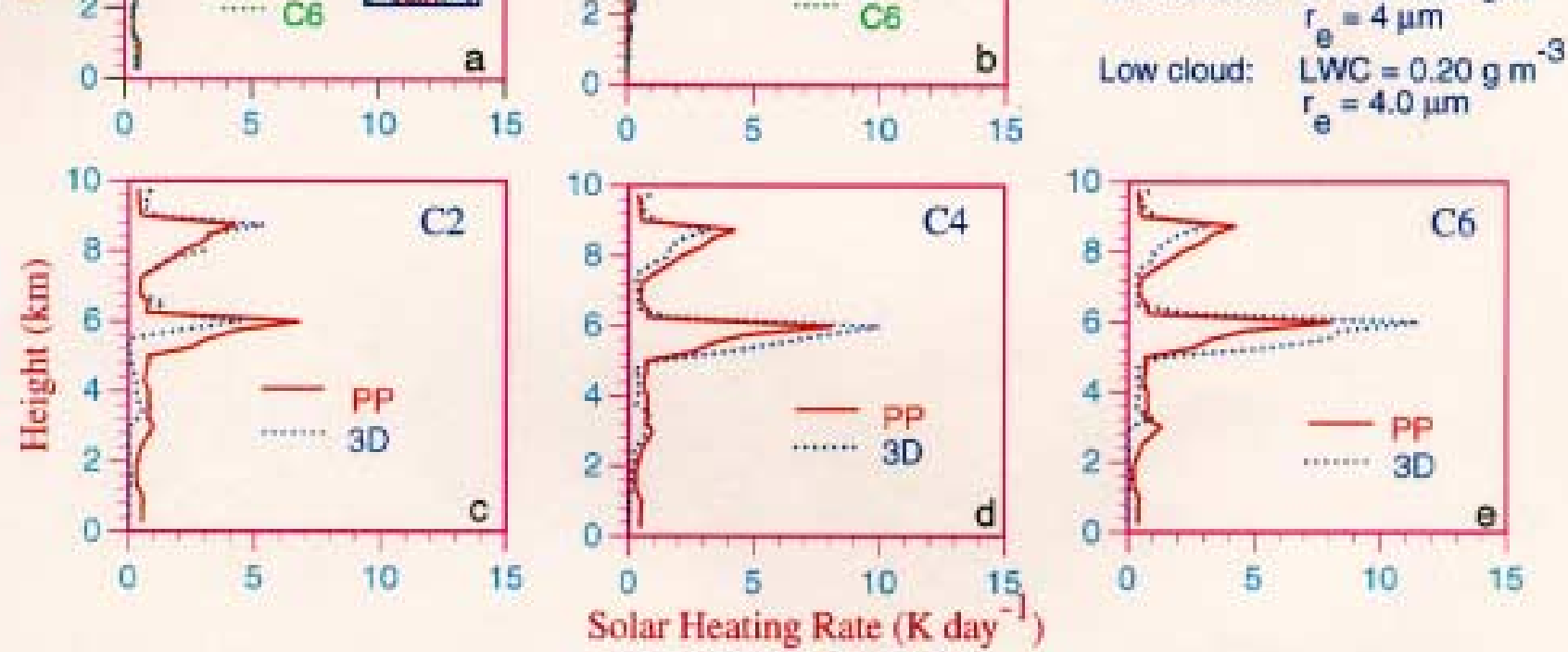

Figure 3. Solar heating rates calculated from (a) the PP method and (b) the 3D model for different cloud overlaps, and comparisons between the PP method (solid) and the 3D model (dashed) for (c) two columns, (d) four columns, and (e) six columns.

less IR cooling at the cloud top and less IR warming at the cloud bottom, as compared to PP clouds as a result of cloud geometry effects (Figure 4c-e). The decrease of IR cooling at the cloud top in the 3D case is at the expense of more IR cooling in clear sky.

Calculations for a single level continuous cloud and broken clouds have been carried out employing the configurations displayed in Figures $2 \mathrm{~d}$ and $2 \mathrm{e}$. While the results computed from the PP model are exactly the same for both cases, those from the 3D model show significant differences. Broken clouds appear to produce more solar heating in the cloud layer because the possibility of photons reflected by one broken cloud and intercepted by another is higher than the PP case. Broken clouds also show more IR cooling at the cloud top. The differences between 3D and PP models in this case are mainly due to the cloud geometry effects.

\section{Conclusion}

A radiative transfer model based on the diffusion approximation approach has been developed to simulate the transfer of radiation in 3D inhomogeneous cirrus clouds. The extinction coefficient, singlescattering albedo, and asymmetry factor are functions of the wavelength and spatial position, and can be parameterized in terms of IWC and mean effective ice crystal size. The delta-function adjustment is 

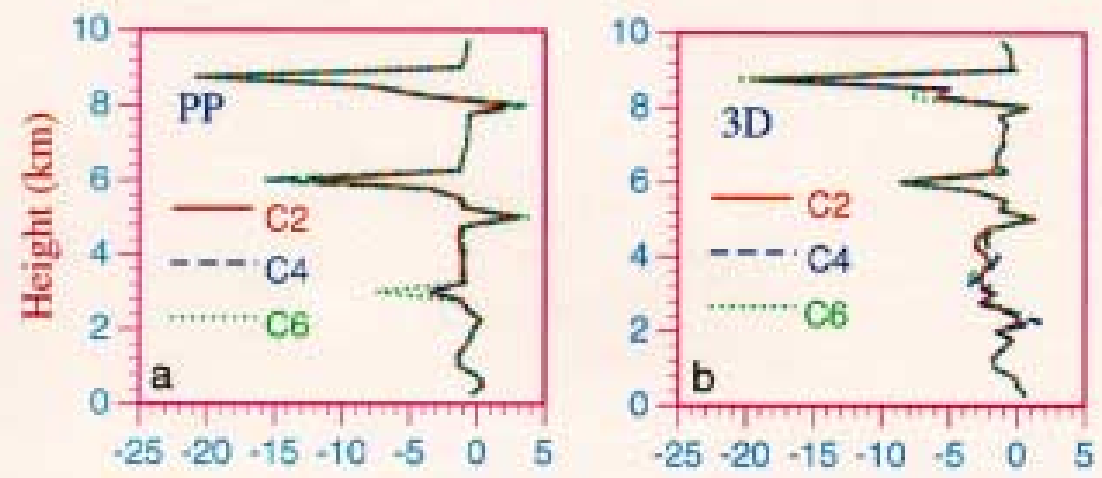

Temperature and $\mathrm{H}_{2} \mathrm{O}$ (standard)

High cloud: $\quad I W C=0.015 \mathrm{~g} \mathrm{~m}^{-3}$ $D_{e}=25 \mu \mathrm{m}$

Middle cloud: $L W C=0.20 \mathrm{~g} \mathrm{~m}^{-3}$

$r_{e}=4 \mu m$

Low cloud: $\quad$ L $W C=0.20 \mathrm{~g} \mathrm{~m}^{-3}$

$r_{e}=4.0 \mu \mathrm{m}$
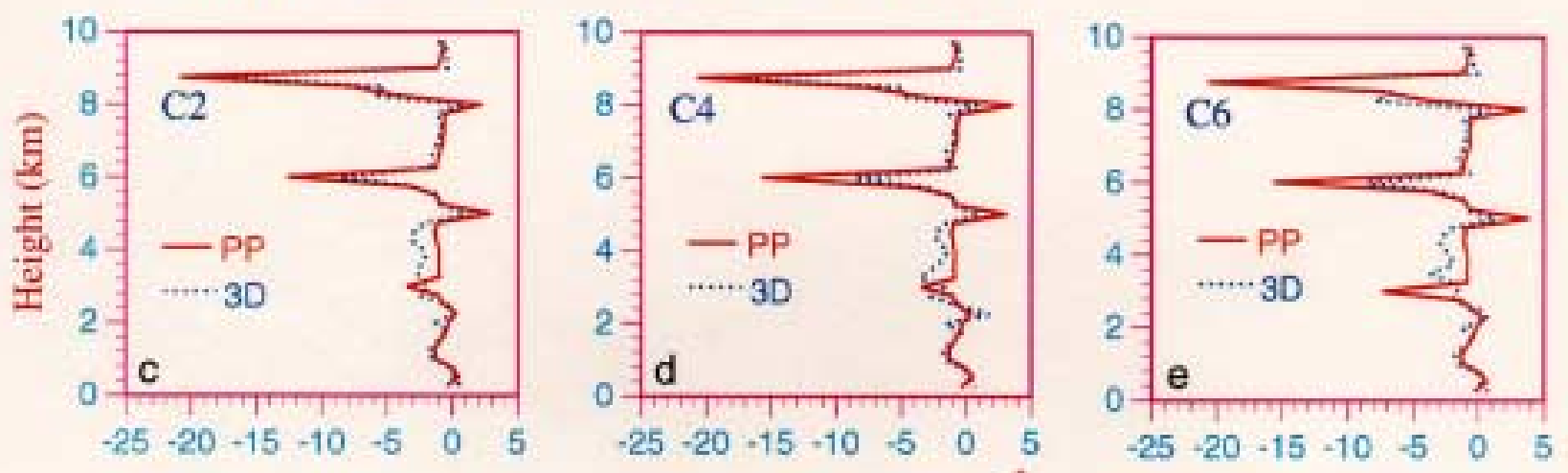

IR Heating Rate $\left(\mathrm{K}_{\text {day }}{ }^{-1}\right)$

Figure 4. Same as Figure 3, except for IR heating rates.

used to account for the forward diffraction peak in the phase function to enhance the computational accuracy. The second-order partial differential transfer equations with proper boundary conditions imposed are solved numerically by using an efficient over-relaxation method. For inhomogeneous cases, upwelling and downwelling fluxes illustrate patterns associated with the extinction coefficient field. Moreover, the cloud inhomogeneity plays an important role in determining heating rate distributions for both solar and IR radiation. We apply the present radiation parameterization for 3D inhomogeneous clouds to climate model studies in terms of domain-averaged heating and cooling rates. Simulation results for a variety of cloud overlaps show that the cloud vertical structure plays a critical role in the determination of domain-averaged solar heating rates. Clouds with random overlap tend to produce more heating than those with maximum overlap. For IR, the differences between 3D and PP models are produced primarily from finite cloud geometry effects. The way in which clouds overlap is of less significance, however. Finally, we show that broken clouds produce more solar heating as well as more IR cooling as compared with the continuous cloud field.

\section{Acknowledgments}

Research supported by U.S. Department of Energy (DOE) Grants DE-FG03-95ER61991 and DE-FG0300ER62904. 


\section{Corresponding Author}

Yu Gu, Department of Atmospheric Sciences, University of California, Los Angeles, Los Angeles, California 90095. Tel: (310) 206-4937; email: gu@ atmos.ucla.edu

\section{References}

Fu, Q., and K. N. Liou, 1993: Parameterization of the radiative properties of cirrus clouds. J. Atmos. Sci., 50, 2008-2025.

Heymsfield, A. J., and L. M. Miloshevich, 1993: Overview of microphysics and state parameter measurements from FIRE-II. Proc. Conf. on FIRE Cirrus Science Results 1993, Breckenridge, Colorado, NASA, 1-4.

Liou, K. N., 1986: Influence of cirrus clouds on weather and climate processes: A global perspective. Mon. Wea. Rev., 114, 1167-1199.

Liou, K. N., 1992: Radiation and cloud processes in the atmosphere: Theory, observation, and modeling. Oxford University Press.

Liou, K. N., and S. C. Ou, 1979: Infrared radiative transfer in finite cloud layers. J. Atmos. Sci., 36, 1985-1996.

Liou, K. N., and N. Rao, 1996: Radiative transfer in cirrus clouds. Part IV: On cloud geometry, inhomogeneity, and absorption. J. Atmos. Sci., 53, 3046-3065.

Ou, S. C., and K. N. Liou, 1982: Generalization of the spherical harmonic method to radiative transfer in multi-dimensional space. J. Quant. Spectrosc. Radiat. Transfer, 28, 271-288.

Ou, S. C., K. N. Liou, Y. Takano, N. X. Rao, Q. Fu, A. J. Heymsfield, L. M. Miloshevich, B. Baum, and S. A. Kinne, 1995: Remote sounding of cirrus cloud optical depths and ice crystal sizes from AVHRR data: Verification using FIRE II IFO measurements. J. Atmos. Sci., 52, 4143-4158. 